\title{
Psychological experiences and quality of life in patients awaiting coronary artery bypass graft
}

\author{
Mousa A. Al-Hassan ${ }^{1 *}$, Samira M. Ahmed ${ }^{2}$, Laila M. Akhu-Zaheya ${ }^{1}$, Jahara N. Hayudini ${ }^{2}$ \\ ${ }^{1}$ Jordan University of Science and Technology Faculty of Nursing, Jordan \\ ${ }^{2}$ Sultan Qaboos University College of Nursing, Oman \\ *Corresponding author E-mail: alhassan@just.edu.jo
}

\begin{abstract}
Background: Coronary artery bypass graft surgery improves quality of life and survival rates. However, prolonged waits for this surgery are inevitable in countries having nationalized healthcare systems. Although no significant decline in the waiting times for this surgery has been demonstrated, recent research in psychological experiences of the patients is lacking

Objectives:

1) To describe psychological experiences, anxiety and uncertainty, and quality of life in Omani patients awaiting coronary artery bypass graft.

2) To examine the significance of sociodemographics, health characteristics and the waiting time in predicting anxiety and uncertainty.

3) To examine the independent effect of anxiety and uncertainty in predicting quality of life of the patients.

Methods: This study used a cross-sectional design. To obtain the required information, two nurses interviewed a convenience sample of hospitalized patients for coronary artery bypass graft $(N=84)$ between May 2011 and June 2012. Three regression analyses were used to identify significant predictors of anxiety, uncertainty and quality of life.

Results: Literate patients and those with infrequent hospital admissions were more likely to experience high levels of anxiety, whereas, patients without myocardial infarction but having hypertension, and those who waited for the surgery 60 days or less were more likely to report greater uncertainty. Exercising regularly, low family monthly income and short duration since diagnosis with coronary artery disease significantly predicted better quality of life

Conclusion: Physiological disruptions and concomitant chronic illnesses have significant effects on the psychological experiences and quality of life of the patients.
\end{abstract}

Keywords: Anxiety, Coronary Artery Bypass Graft, Quality Of Life, Uncertainty, Waiting Time.

\section{Introduction}

Coronary artery bypass graft $(\mathrm{CABG})$ is an important surgical intervention for patients experiencing coronary artery diseases (CADs). This surgery restores blood supply to the heart using arteries or veins from other parts of the body to bypass the narrowed coronary arteries (Fitzsimons et al. 2003). It relieves angina, prolongs life, and improves quality of life. Although this surgical revascularization at times deemed acceptable to the surgeons improves survival rate (Sobolev et al. 2008), prolonged waits are inevitable, particularly in countries having nationalised health care systems, because the demand on the CABG exceeds available resources (Sampalis et al. 2001, McCormick et al. 2006). Patients with prolonged waits may experience unstable angina, myocardial infarction or cardiac death (Cesena et al. 2004, Rexius et al. 2005). Long waits for CABG were also found to increase the risk for deaths before surgery (Sobolev et al. 2008).

Psychologically, the patients awaiting CABG may experience anxiety (Fitzsimons et al. 2003), uncertainty (Screeche-Powell \& Owen 2003, McCormick et al. 2005) and worsened quality of life (QOL) (Sampalis et al. 2001). However, studies investigating psychological experience of the patients awaiting CABG are most often descriptive in that they lacked information about factors contributing to psychological adverse events and QOL that the patients may experience. Although no significant decline in the waiting period across time has been observed (NHS strategic health authority 2013), recent studies on psychological experiences of patients awaiting CABG are lacking. Moreover, no study describing psychological experiences of patients awaiting CABG, in Oman and other Arab states, has been reported. Information about the psychological experience is necessary to build nursing assessment guidelines to identify those at risk for psychological adverse events and deteriorated QOL, and intervention protocols that would help patients to cope with problems of long wait.

Oman is an Arab Gulf state where Islam dominates its culture that is characterised by an extended family structure. Arab Moslems believe that wellness and illness is God's will. They value strong social bonds among family members, regard parents and elderly with more respect and may use Islam religious practices (prayers) to cope with stressful situations. In the past few decades, Oman has experienced rapid modernization and acculturation (Al-Adawi 2006), besides, it has experienced an economic development, because of oil money that results in lifestyles changes and emergence of diseases of affluence (Al-Sharbati et al. 2003) such as increasing the risk factors for CADs (Al Riyami \& Afifi 2003). The health care system in Oman is community based which provides free health services to both Omanis and expatriates. Although the Omani heath care system has shown rapid progress and development in the recent years (Al-Adawi, 2006), it lacks formal 
patient education programs and follow-up care at home that may limit provision of CABG-related information to the patients.

The major purpose of this study was to assess psychological experiences and QOL in Omani patients awaiting CABG. Specifically, this study attempted to a) describe waiting time, anxiety, uncertainty and QOL in Omani patients awaiting CABG, b) examine the significance of sociodemographics, health characteristics and the waiting time for CABG in predicting anxiety and uncertainty, and c) examine the independent effect of anxiety and uncertainty in predicting QOL.

\section{Background}

A waiting time refers to the length of time between when the patient is placed on a waiting list for CABG and when the surgery is eventually performed. The median of the waiting times, in Western societies, ranges from 55 days to 6 months (Koomen et al. 2001, Rexius et al. 2005), with a reasonable portion of the patients is waiting longer than 12 months (Cesena et al. 2004). With delayed CABG, significant mortality (Rexius et al. 2005) and morbidity (Fitzsimons et al. 01/2000) may occur. The most prominent symptoms of the patients are anxiety, pain, fatigue and uncertainty (McCormick et al. 2006), physical incapacity (Fitzsimons et al. 2003) and fear (Koivula et al. 2001).

It seems that anxiety is a feature of patients awaiting CABG. A multi-method study on randomly selected 70 patients awaiting cardiac surgery reported that the patients had experienced high level of anxiety that was consistent 2-4 weeks, 6 months and one year of waiting (Fitzsimons et al. 2003). A moderate level of anxiety was also reported by patients awaiting coronary angiography (De Jong-Watt \& Arthur 2004). However, inconsistent findings were reported about sources of anxiety. Data of a qualitative study indicated that pain, uncertainty, physical incapacity, and an impending surgery were sources of anxiety (Fitzsimons et al. 2003). In this patient population, anxiety was found to associate symptoms frequency and symptoms distress (McCormick et al. 2006) and increasing angina (Fitzsimons et al. 2003). However, another study demonstrated that low levels of anginal pain significantly predicted high anxiety levels (Koviula et al. 2001). On the other hand, no significant relationship between anxiety and waiting time for CABG was found (McCormick et al. 2006).

Mishel (1990) has defined uncertainty as "the inability to determine the meaning of illness-related events" (P. 25). Two studies (Screeche-Powell \& Owen, 2003, McCormick et al. 2006) demonstrated that patients awaiting CABG were experiencing moderate levels of uncertainty. Long waits could create psychological disturbances including uncertainty (McCormick et al. 2005) in which timing of treatment plans is a source of uncertainty (Lindsay et al. 1997, Staples \& Jeffery 1997). The patients are uncertain about when the operation would take place which is perceived as a major difficulty for the patients and their families (Fitzsimons et al 2000).

Patients awaiting CABG may also experience poor quality of life Of the patients who were placed on a managed waiting list for more than 6 weeks, 87\% reported deteriorated QOL (Teo et al. 1998). Findings of a prospective study revealed that QOL decreased with increased waiting time; patients who waited longer than 97 days reported decreased physical and social functioning than those who waited a shorter period (Sampalis et al. 2001). In another prospective study, significant reduction in physical and social functioning was observed between 6 months and one year of waiting for the CABG (Fitzsimons et al. 2000). The Seattle Angina Questionnaire, a health-related QOL measure, was introduced to 40 patients with CADs awaiting coronary angiography at time of placement on the waiting list and one week before the procedure (De Jong-Watt \& Arthur, 2004). The researchers found that the patients reported decreased physical functioning in the later observation compared to the first.

Deterioration of QOL of patients awaiting CABG may be related to the stressful nature of the waiting time. Patients awaiting
CABG were found to be dissatisfied with their health status, and 86\% experienced stress (Jonsdottir \& Baldursdottir 1998). Anxiety and uncertainty were found to significantly correlate with deteriorated functional capacity (McCormick et al. 2006), and greater uncertainty significantly predicted poor QOL in patients awaiting CABG (Staples \& Jeffery 1997).

\section{Methods}

\subsection{Design and sample}

A cross-sectional design was used. Potential participants were Omani patients awaiting first-time CABG at a Ministry of Health affiliated major hospital in Muscat area, Oman. All Omani patients who were admitted to the intermediate cardiac unit of the selected hospital for the CABG between May First, 2011 and June 30, 2012 were potential participants. The patients were included, in the study, if they were a) 30 years of age or older, b) on the waiting list and c) waited at home for 4 weeks or more. Patients with combined $\mathrm{CABG}$ and valve replacement and those having psychiatric problems were excluded.

\subsection{Ethical consideration}

The study was approved by a university research committee and the ethics and scientific research committee of the selected hospital. An informed verbal consent was obtained from each participant, where an adult family member of first degree relationship with the patient witnessed the consenting process. This consenting process was used to overcome the illiteracy problem.

\subsection{Procedure}

Two bachelor-prepared nurses working in the intermediate cardiac unit of the selected hospital collected the data using a structured interview. The interview took approximately 30 minutes to complete. Once the approvals were secured, the primary researcher informed the head nurse of the unit about the study. Then, he explained to the interviewers the study's purposes, significance and data collection method. Usually the patients are admitted two days before the CABG, during which the data were collected. The interviewers approached the unit, in the working days, looking for newly admitted patients as potential participants. They reviewed files of eligible patients, and extracted the required health information, then, approached and informed the patients about the study purpose, significance and data collection method, that participation was voluntary and invited them to participate. Finally, the reviewers did data collection from those who accepted participation.

\subsection{Measurements}

The researchers used four instruments. First, the sociodemographics and health characteristics tool which includes questions about age, gender, marital status, income, living status, working status and number of years spent in formal education. The tool also sought information about smoking, regular exercising, left ventricular ejection fraction, number of occluded coronary arteries, number of hospital admissions because of cardiac events, chest pain intensity measured on the Visual Analogue Scale, time since diagnosis with CADs and whether or not patients have myocardial infarction, hypertension and diabetes mellitus. The waiting time was measured by asking each participant "How many days did it take from the time you were placed in the waiting list for CABG to the time of hospital admission to undergo the surgery"

Second, the visual analogue scale (VAS) was used to measure anxiety. The VAS is a $10-\mathrm{cm}$ horizontal line where extremely anxious marks the right end and not at all anxious marks the left end. The validity of the VAS was established (Benotsch et al. 2000). In recent studies, the VAS has been found to highly corre- 
late with the Dental Anxiety scale $(r=0.57, \mathrm{P}=.00)$ (Facco et al. 2011) and the Hospital Anxiety and Depression Scale ( $\mathrm{r}=.74, \mathrm{P}=$ .00) (Williams et al. 2010). The participants were asked to mark a point on the VAS line indicating how much anxiety they felt during the waiting period. Anxiety of the patients was the distance between the not at all anxious end and the patient's mark.

Third, the Mishel Uncertainty in Illness Scale (MUIS) (Mishel, 1997) was used to measure uncertainty. This scale has 23 items with a five-point Likert scale responses ranging from "1" "strongly disagree" to "5" strongly agree. The total score was obtained by adding the responses of all items which ranges from 23 to 115 with higher scores indicating greater perceived uncertainty. Internal consistency reliabilities of .89 and .91 were reported for the MUIS (Eastwood et al. 2008). Once the permission to use the MUIS was secured, the scale was translated to Arabic language through translation and back-translation process. A panel of 4 persons who are competent in English and Arabic languages participated in the translation process. Two persons, one expert in health sciences and one lay person translated the scale from English into Arabic language. The other two did back-translation. Any discrepancy between the 2 versions was resolved based on the panel's suggestion. In this study, the Arabic version of the MUIS showed internal consistency reliability of $\alpha=.72$, and the content validity was supported by three master-prepared cardiac nurses.

Finally, the Medical Outcomes Study Short Form (MOS SF-36) was used to measure QOL. This scale includes 36 questions reflecting the QOL in eight different dimensions: bodily pain $(2$ items), social functioning (2 items), vitality (4 items), emotional well-being (5 items), general health (5 items), physical functioning (10 items), role limitations due to physical health (4 items), and role limitation due to emotional health (3 items). Each item is scored from 0 to 100 , and summation of the 36 items' scores is needed to obtain the QOL score, with greater score indicating better QOL. The SF-36 scale was found to be valid and reliable ((McHorney et al. 1994).

In this study, the researchers used the Arabic version of the SF-36 which was validated in the Saudi Arabia (Coons et al. 1998) and Lebanon (Sabbah et al. 2003). In Morocco, acceptable levels of the internal consistency $(.70)$ and test-retest reliability $(.80)$ of the Arabic version of the SF-36 were reported (Khoudri et al. 2007). The same authors confirmed the construct validity of this questionnaire. The internal consistency reliability of the SF-36, in this study, was .88 .

\subsection{Data Analysis}

Data were analyzed using the Statistical Package for Social Science (SPSS) version 17.0. Descriptive statistics (frequencies, percentages, mean (SD) were employed to describe the sample and study variables. The researchers used multiple regression analyses to examine the significance of sociodemographics, health characteristics and the waiting time in predicting anxiety and uncertainty, and independent effect of anxiety and uncertainty in predicting QOL. For anxiety and uncertainty two regression models were conducted. First, sociodemographics and health characteristics were introduced. In the second regression model, the waiting time was added. Three regression models were conducted for QOL Sociodemographics and health characteristics were introduced first, then, the waiting time and finally anxiety and uncertainty were introduced to the regression models two and three respectively. Because of skewness, some variables, educational level, the family monthly income and waiting period, were dichotomized based on the median. The statistical significance was set at 0.05 .

\section{Results}

\subsection{Sample characteristics}

Between May One, 2011 and June 30, 2012, 92 patients met the inclusion criteria, of who 8 refused to participate. Therefore, 84 patients composed the study sample. As table one shows, the majority was male, older adult, married and lived with their families, and one-fourth was active workers. The median of family monthly income of the participants was 560.5 Omani Riyals (1455 \$ US), $58.3 \%$ of the sample was illiterate (Median $=0$ years of formal education), several patients were active smoker and almost onethird was regular exercisers. The mean time since diagnosis with CADs was 3 months, during which the participants reported a moderately high chest pain intensity, were admitted to the hospital 0-15 times. They demonstrated low level of left ventricular ejection fraction and one to 3 occluded coronary arteries. Nine patients reported having myocardial infarction, however, the majority reported having hypertension and about one-third was having diabetes mellitus. The sample waited for the CABG almost 3 months, with $28.6 \%$ was waiting more than 3 months, and 44 patients $(52.4 \%$ ) waited 2 months or less (Median $=60$ days) (see Table $1)$.

Table 1: Sociodemographics and Health Characteristics of the Sample

\begin{tabular}{|c|c|c|c|c|}
\hline Characteristic & $\mathrm{F}$ & $\%$ & $\mathrm{M}(\mathrm{SD})$ & Range \\
\hline \multicolumn{5}{|l|}{ Gender } \\
\hline Male & 65 & 77.4 & - & - \\
\hline Female & 19 & 22.6 & & \\
\hline \multicolumn{5}{|l|}{ Marital status } \\
\hline Single & 3 & 3.6 & & \\
\hline Married & 79 & 94 & & \\
\hline Widowed & 2 & 2.4 & & \\
\hline \multicolumn{5}{|l|}{ Living status } \\
\hline Alone & 1 & 3.5 & & \\
\hline With my family & 76 & 90.5 & & \\
\hline With a sibling & 5 & 6.0 & & \\
\hline \multicolumn{5}{|l|}{ Working status } \\
\hline Currently working & 22 & 26.2 & & \\
\hline Retired & 21 & 25 & & \\
\hline Currently not working & 41 & 48.8 & & \\
\hline \multicolumn{5}{|l|}{ Smoking status } \\
\hline Currently smoking & 6 & 71 & & \\
\hline Never smoked & $\begin{array}{l}6 \\
56\end{array}$ & $\begin{array}{l}7.1 \\
66.7\end{array}$ & - & - \\
\hline Quit smoking & 13 & 15.5 & & \\
\hline $\begin{array}{l}\text { Quit smoking because of } \\
\text { health reasons }\end{array}$ & $\begin{array}{l}13 \\
9\end{array}$ & $\begin{array}{l}15.3 \\
10.7\end{array}$ & & \\
\hline \multicolumn{5}{|l|}{ Regular exercisers } \\
\hline Yes & 27 & 32.1 & - & - \\
\hline No & 57 & 67.9 & & \\
\hline \multicolumn{5}{|l|}{ Having myocardial infarction } \\
\hline Yes & 9 & 10.7 & & \\
\hline No & 75 & 89.3 & & \\
\hline \multicolumn{5}{|l|}{ Having diabetes mellitus } \\
\hline Yes & 29 & 34.5 & & \\
\hline No & 55 & 65.6 & & \\
\hline \multicolumn{5}{|l|}{ Having hypertension } \\
\hline Yes & 58 & 69.0 & & \\
\hline No & 26 & 31.0 & & \\
\hline Age & & & $58.3(10.4)$ & $37-80$ \\
\hline Family monthly income & & & $597(665.5)$ & $\begin{array}{l}50- \\
6000\end{array}$ \\
\hline $\begin{array}{l}\text { Number of years spent in formal } \\
\text { education }\end{array}$ & & & $3.2(4.3)$ & $0-14$ \\
\hline Time since diagnosis with CADs ${ }^{\mathrm{a}}$ & - & - & $3.05(1.9)$ & $0-10$ \\
\hline Intensity of chest pain & - & - & $7.0(2.03)$ & $0-10$ \\
\hline Number of hospital admissions & - & - & $2.13(2.4)$ & $0-15$ \\
\hline Left ventricular ejection fraction & - & - & $46.8(12.7)$ & $20-75$ \\
\hline $\begin{array}{l}\text { Number of occluded coronary } \\
\text { arteries }\end{array}$ & - & - & $2.8(.34)$ & $1-3$ \\
\hline Waiting time for $\mathrm{CABG}^{\mathrm{b}}$ & - & - & $85.7(69.6)$ & $7-364$ \\
\hline
\end{tabular}

A. $\mathrm{CADs}=$ Coronary Artery Diseases

B. $\mathrm{CABG}=$ Coronary Artery Bypass Graft

\subsection{Psychological experiences and QOL of the sample}

As Table two shows, the sample reported moderate levels of anxiety, uncertainty and poor QOL, with $32 \%$ of the patients reported high levels of anxiety (8-10 on the VAS), $42.2 \%$ uncer- 
tainty greater than the median (68.9) and 82\% scored the SF-36 below 50 .

Table 2: Anxiety, Uncertainty, and QOL of the Sample

\begin{tabular}{lll}
\hline Variables & $\mathrm{M}(\mathrm{SD})$ & Potential range \\
\hline Anxiety & $6.0(2.5)$ & $0-10$ \\
Uncertainty & $68.8(8.4)$ & $23-115$ \\
Quality of life & $40.6(18.8)$ & $0-100$ \\
\hline
\end{tabular}

On the bivariate level, waiting time for $\mathrm{CABG}$ had no significant effect on anxiety $(\mathrm{P}=.08)$ and $\mathrm{QOL}(\mathrm{P}=.15)$, however, patients who waited 60 days or less reported significantly $(t=2.1, d f=82$, $\mathrm{P}=.04,955 \mathrm{CI}=.17-7.3)$ higher levels of uncertainty $(\mathrm{M}=70.6$, $\mathrm{SD}=8.1)$ than those who waited more than two months $(\mathrm{M}=$ $66.8, \mathrm{SD}=8.4)$. Patients with diabetes mellitus reported significantly $(\mathrm{t}=-2.4, \mathrm{df}=82, \mathrm{P}=.02,95 \% \mathrm{CI}=-8.3--.8)$ lower uncertainty $(\mathrm{M}=65.8, \mathrm{SD}=9.6)$ than those who were free from diabetes $(\mathrm{M}=70.4, \mathrm{SD}=7.3)$. Also, patients with hypertension reported significantly $(\mathrm{t}=2.9, \mathrm{df}=82, \mathrm{P}=.01,95 \% \mathrm{CI}=1.7-9.3)$ higher uncertainty levels $(\mathrm{M}=70.5, \mathrm{SD}=6.6)$ than those without hypertension $(\mathrm{M}=64.9, \mathrm{SD}=10.6)$.

On the other hand, regular exercisers reported significantly $(\mathrm{t}=$ $2.0, \mathrm{df}=82, \mathrm{P}=.04,95 \% \mathrm{CI}=.06-11.7)$ better QOL $(\mathrm{M}=44.6$, $\mathrm{SD}=14.4)$ than non-exercisers $(\mathrm{M} 38.7, \mathrm{SD}=11.5)$. Also, $\mathrm{QOL}$ negatively and significantly associated time since diagnosis with
CADs $(r=-.30, P=.00)$ and chest pain intensity $(r=-.23, P=$ $.03)$. Anxiety significantly and positively correlated with uncertainty $(\mathrm{r}=.35, \mathrm{P}=.00)$, but negatively with $\mathrm{QOL}(\mathrm{r}=-.40, \mathrm{P}=$ $.00)$. Other sociodemographics and health characteristics did not significantly affect anxiety, uncertainty or QOL.

\subsection{Significant predictors of anxiety, uncertainty and QOL.}

As Table 3 shows, the model of sociodemographics and health characteristics significantly predicted anxiety $(\mathrm{F}=2.0, \mathrm{P}=.02)$ explaining $16 \%$ of the variance. Addition of the waiting time decreased the variance in anxiety to $15 \%$, although the model was statistically significant $(\mathrm{F}=1.6, \mathrm{P}=.03)$. The regression analysis indicated that illiterate patients and those with increased number of hospital admissions were more likely to experience less anxiety. In the second regression analysis, sociodemographics and health characteristics significantly predicted uncertainty $(\mathrm{F}=2.2, \mathrm{P}=$ .01 ), explaining $18 \%$ of the variance. In model two, addition of the waiting time significantly increased the explained variance in uncertainty $4 \%$. Patients awaiting $\mathrm{CABG}$ who were free from myocardial infarction, but having hypertension, and waited 60 days or less reported greater uncertainty.

Table 3: Significant Predictors of Anxiety and Uncertainty

\begin{tabular}{|c|c|c|c|c|c|c|c|c|}
\hline \multirow[b]{2}{*}{$\begin{array}{l}\text { Model } \\
\text { no. }\end{array}$} & \multicolumn{4}{|l|}{ Anxiety } & \multicolumn{4}{|l|}{ Uncertainty } \\
\hline & Variables & Adjusted $\mathrm{R}^{2}$ & $\beta$ & $\mathrm{P}$ & Variables & Adjusted $\mathrm{R}^{2}$ & $\beta$ & $\mathrm{P}$ \\
\hline \multirow{8}{*}{2} & $\begin{array}{l}\text { Sociodemographics and health char- } \\
\text { acteristics }\end{array}$ & .16 & & & $\begin{array}{l}\text { Sociodemographics and health } \\
\text { characteristics }\end{array}$ & .18 & & \\
\hline & Formal education & & .35 & .02 & Having myocardial infarction & & -.32 & .00 \\
\hline & Number of hospital admissions & & .31 & .01 & Having Hypertension & & .30 & .00 \\
\hline & Time since diagnosis with CADs & & .24 & .04 & & & & \\
\hline & $\begin{array}{l}\text { Sociodemographics, health character- } \\
\text { istics and waiting time }\end{array}$ & .15 & & & $\begin{array}{l}\text { Sociodemographics, health charac- } \\
\text { teristics and waiting time }\end{array}$ & .22 & & \\
\hline & Formal education & & .35 & .03 & Having myocardial infarction & & -.32 & .00 \\
\hline & No. of hospital admissions & & -.30 & .01 & Having hypertension & & .30 & .00 \\
\hline & Waiting time & & - & .38 & Waiting time & & -.23 & .04 \\
\hline
\end{tabular}

Significant predictors of QOL are shown in Table 4. The regression analysis indicated that model one was statistically significant ( $\mathrm{F}=2.3, \mathrm{P}=.01)$ in predicting QOL explaining $20 \%$ of the variance. Addition of the waiting time did not increase the variance in QOL, although the model was statistically significant $(\mathrm{F}=2.3, \mathrm{P}=$ $.01)$. In the third model, addition of anxiety and uncertainty sig- nificantly increased the variance in QOL $6 \%(\mathrm{~F}=2.6, \mathrm{P}=.00)$. Patients awaiting CABG who were exercising regularly, had low family monthly income, and reported less time since diagnosis with CADs experienced better QOL. However, anxiety $(\mathrm{P}=.20)$ and uncertainty $(\mathrm{P}=.07)$ did not significantly predict $\mathrm{QOL}$.

Table 4: Significant Predictors of QOL

\begin{tabular}{|c|c|c|c|c|}
\hline Model No. & Variables & Adjusted $\mathrm{R}^{2}$ & $\mathrm{~B}$ & $\mathrm{P}$ \\
\hline \multirow[t]{4}{*}{1.} & Sociodemographics and health characteristics & .20 & & \\
\hline & Family monthly income & & -.32 & .00 \\
\hline & Time since diagnosis with CADs* & & -.30 & .01 \\
\hline & Exercising regularly & & .23 & .03 \\
\hline \multirow[t]{2}{*}{2.} & $\begin{array}{l}\text { Sociodemographics, health characteristics and waiting time } \\
\text { Family monthly income }\end{array}$ & .20 & & \\
\hline & Time since diagnosis with CADs* & & $\begin{array}{l}-.35 \\
-.29\end{array}$ & $\begin{array}{l}.00 \\
.01\end{array}$ \\
\hline \multirow[t]{4}{*}{3.} & $\begin{array}{l}\text { Sociodemographics, health characteristics, waiting time, and anxiety and un- } \\
\text { certainty }\end{array}$ & .26 & & \\
\hline & Income & & -.31 & .00 \\
\hline & Exercising & & .24 & .03 \\
\hline & Time since diagnosis with CADs* & & -.23 & .04 \\
\hline
\end{tabular}

$*$ CADs $=$ Coronary Artery Diseases

\section{Discussion}

Almost half of this sample waited for CABG more than 2 months with a reasonable percentage was waiting more than 90 days. This period is clinically significant since the majority of cardiac related events occur in the first 30 days of waiting (Koomen et al. 2001) In the Western culture, inconsistent findings were reported about the waiting time for CABG. In a sample of Scandinavian cardiac patients, the median of waiting period was 55 days (Rexius et al. 2005), whereas, longer waiting times were reported by a Nieuwgein sample (Median $=100$ days) $($ Koomen et al. 2001) and Candian patients (Median $=97$ days) $($ McCormick et al. 2006) . The shorter waiting time reported by this sample of Omani patients compared to that reported in the Western culture may be related to differences in measuring the waiting time. In the current study, the perceived waiting period was measured which may be influenced by a recall bias. 
The participants reported moderate levels of anxiety and uncertainty during the waiting time. Similar findings were reported by cardiac patients in Northern Ireland (Fitzsimons et al. 2003) and Canada (McCormick et al. 2006). In a qualitative study, ScreechePowell and Owen (2003) found that patients awaiting CABG experienced fears about specific aspects of the CABG. In another study (De Jong-Watt \& Arthur, 2004), a moderate level of anxiety was reported by patients awaiting coronary angiography.

In the current study, patients with greater anxiety were more likely to be literate and reported decreased number of hospital admissions. It may be that literate patients were more informed about various issues about $\mathrm{CABG}$, particularly they are able to read written materials, therefore, they raised more worries about such issues. However, they might have little opportunity to receive information about consequences of the CABG because of infrequent admissions to the hospital. In the Western culture, however, different correlates to anxiety, in the waiting time, were reported such as angina ((Fitzsimons et al. 2003), uncertainty (ScreechePowell \& and Owen 2003), age, sex and education (Koivula et al. 2001). In this sample, the waiting time was not a significant predictor of anxiety. This finding may be explained by the study's small sample size. In a sample of Canadian cardiac patient the waiting time for $\mathrm{CABG}$ did not significantly associate anxiety (McCormick et al. 2006).

The current sample reported a moderate level of uncertainty. In three qualitative studies (Fitzsimons et al. 2000, Screeche-Powell \& Owen, 2003, McCormick et al. 2005), that were conducted in the Western culture, uncertainty was a major theme patients awaiting CABG did raise. The level of uncertainty the patients, in the current study, reported $(68.8, \mathrm{SD}=8.4)$ is higher than what Canadian patients reported $(\mathrm{M}=58.4, \mathrm{SD}=13.5$ ) (McCormick et al 2006). The difference in uncertainty may be related to that the Omani patients lack information about the CABG, as the Omani health care system lack formal patient education programs and follow-up care after discharge. The factors, waiting for CABG 60 days or less, having hypertension but not myocardial infarction, significantly predicted greater uncertainty. In a qualitative study, however, it was concluded that the length of the waiting time could generate significant uncertainty (McCormick et al. 2005) Uncertainty that the patients awaiting CABG do experience may be related to that the patients were not given adequate information about the surgery (Fitzsimons et al. 2000). Data describing correlates of uncertainty are lacking. In one study, symptoms distress did not significantly affect uncertainty (McCormick et al. 2006 While having myocardial infarction predicted less uncertainty, in the current study, having hypertension significantly predicted greater uncertainty. These findings are perplexing and difficult to explain. However, the patients with myocardial infarction might receive more information about CABG surgery, during their clinic visit, compared to those with hypertension.

This sample of Omani patients reported experiencing poor level of QOL. In a Canadian sample of patients awaiting CABG, $87.5 \%$ reported deterioration in their QOL since being placed on the waiting list (Teo et al. 1998), and those patients who waited more than 97 days reported reduced physical functioning, vitality, social functioning and general health (Sampalis et al. 2001). Regression analysis indicated that Omani patients awaiting CABG who had a family monthly income of less than 560.5 Omani Riyals, were exercising regularly and reported decreased time since diagnosis with CADs were more likely to experience better QOL. The negative association between family monthly income and QOL may be explained by the notion that costs of the CABG are not a concern to the Omani patients since the Omani health care system provides free healthcare services to all Omani citizens. However, those with high income might experience worries about their sources of their income that may negatively affect the patients' QOL, particularly long waits for CABG were more likely to associate not returning to work (Sampalis et al. 2001). It is well known that exercising regularly improves the physiological and psychological health of the patients. Experiencing CADs for a short period before CABG decreases the opportunity of developing adverse cardiac-related events that worsen QOL. The majority of patients awaiting CABG were found to be dissatisfied with their health status and experiencing fatigue, shortness of breath, chest pain and anxiety (Jonsdottir \& Baldursdottir 1998) that may negatively affect the patients' life. Uncertainty did not significantly predicted QOL, in the current study. This finding could be explained by the small sample size. In the Western culture, negative associations between uncertainty, and functional status of the patients and symptoms frequency and distress were demonstrated (McCormick et al. 2006).

\section{Implications and recommendations}

Nurse leaders should work on developing assessment protocols that focus on physiological and psychological experiences of patients awaiting CABG to identify those at risk for developing anxiety, uncertainty and deteriorated QOL. Culturally-sensitive assessment protocols may also be required to identify culturespecific factors that contribute to psychological changes and deteriorated quality of life.

Practicing nurses should initiate interventions to control concomitant illnesses that associate CADs, promote regular exercising and manage cardiac-related events that may occur in the waiting time for CABG. Replication of this study using a prospective design on a randomly selected sample of patients awaiting CABG from different settings is required to investigate psychological events and quality of life that patients awaiting CABG may experience. Identification of significant predictors of such psychological events and quality of life using more valid instruments is also essential for quality nursing care.

\section{Limitations}

The current study's findings should be interpreted in light of the following limitations. A small sample size that was non-randomly selected from one hospital limits the generalizability of the study. The small sample size reduces the power of the study to detect significant relationships. Retrospective measurement of the study variables may bring a recall bias that negatively affects the internal validity of the results.

\section{Conclusion}

In brief, patients awaiting CABG experience moderate levels of anxiety and uncertainty, and poor QOL. Anxiety was related to literacy and decreased number of hospital admissions, whereas, not having myocardial infarction but experiencing hypertension and short waiting time for CABG predicted greater uncertainty. It seems that the physiological aspects, exercising regularly and experiencing CADs, are influential in the patients' QOL, during the waiting time.

Funding: This study was funded by Sultan Qaboos University, Deanship of Research. Grant Number: IG/CN/ACCNL11/01.

\section{References}

[1] Al-Adawi S (2006) Emergence of diseases of affluence in Oman. Sultan Qaboos University Medical Journal 6, 3-9.

[2] Al Riyami AA \& Afifi M (2003) Clustering of cardiovascular risk factors among Omani adults. Eastern Mediterranean Health Journal 9, 893-903.

[3] Al-Sharbati MM, Al-Lawatiya S, Al-Adawi S, Martin R \& AlHussaini A (2003) Urbanization, culture and hyperactivity: Naturalistic observation in Omani schoolgirls. Women's Health \& Urban Life 2, 43-60.

[4] Benotsch EG, Lutgendorf SK, Watson D, Fick LG \& Lang E V (2000) Rapid anxiety assessment in medical patients: Evidence for the validity of the verbal anxiety ratings. Annals of Behavioral Medicine 22, 199-203. 
[5] Cesena FH, Favarato D, Cesar LA, de Oliveira SA \& da Luz PL (2004) Cardiac complications during waiting for elective coronary artery bypass graft surgery: incidence, temporal distribution and predictive factors. European Journal of Cardio-thoracic Surgery 25, 196202.

[6] Coons SJ, Alabdulmohsin SA, Draugalis JL \& Hays RD (1998) Reliability of an Arabic version of the RAND36 health survey and its equivalence to the US-English. Medical care 36, 428-432.

[7] De Jong-Watt W \& Arthur H (2004). Anxiety and health-related quality of life in patients awaiting elective coronary angiography. Heart and Lung 33, 237-248.

[8] Eastwood J, Doering L, Roper J \& Hayes R D (2008). Uncertainty and health Related quality of life 1 year after coronary angiography. American Journal of Critical Care 7, 232-242.

[9] Facco E, Zanette G, Favero L, Bacci C, Sivolella S, Cavallin F \& Manani G (2011) Toward the validation of visual analogue scale for anxiety. Anesthesia Progress 58, 8-13. DOI: 10.23344/00033006.1.8.

[10] Fitzsimons D, Parahoo K, Stringer M. (2000) Waiting for coronary artery bypass surgery a qualitative analysis. Journal of Advanced Nursing 32, 1243-1252.

[11] Fitzsimons D, Richardson S\& Scott ME (01/2000). Prospective study of clinical and functional status in patients awaiting coronary artery bypass surgery. Coronary Health Care 4, 117-122. DOI: 10. 1054/che.2000.0082.

[12] Fitzsimons D, Parahoo K, Richardson S \& Stringer M (2003) Patient anxiety while on a waiting list for coronary artery bypass surgery: a qualitative and quantitative analysis. Heart and Lung 32, 23-31.

[13] Jonsdottir H \& Baldursdottir L (1998) the experience of people awaiting coronary artery bypass graft surgery: the Icelandic experience. Journal of Advanced Nursing 27, 68-74.

[14] Khourdi I, Zeggwagh A, Abidi K, Madani N \& Abouqal R (2007) Measurement properties of the short form 36 health-related quality of life after intensive care in Morocco. Acta Anaesthesiologica Scandinavica 51, 189-197.

[15] Koivula M, Paunonen-Ilmonen M, Tarkka M, Tarkka MT \& Laippala P (2001) Fear and anxiety in patients awaiting coronary artery bypass grafting. Heart and Lung 30, 302-311.

[16] Koomen EM, Hutten BA, Kelder JC, Redekop W, Tijssen JG \& Kingma J (2001) Morbidity and mortality in patients waiting for coronary artery bypass surgery. European Journal of Cardio-Thoracic Surgery 19, 260-265.

[17] Lindsay, P., Sherrad, H., Bikerton, I., Doucette, P., Harkness, C., \& Morin, J. (1997). Educational and support needs of patients and their families awaiting cardiac surgery. Heart and Lung, 26, 458-465.

[18] McCormick, K. M., McClement, S., Naimark, B. J. (2005). A qualitative analysis of the experience of uncertainty while awaiting coronary artery bypass surgery. Canadian Journal of Cardiovascular Nursing, 5, 10-22

[19] McCormick K, Naimark B \& Tate R (2006) Uncertainty, symptom distress, anxiety, and functional status in patients awaiting coronary artery bypass surgery. Heart Lung 35, 34-45.

[20] McHorney CA, Ware JE, Lu JF \& Sherbourne CD (1994) The MOS 36-item Short Form Health Survey (SF-36) III. Tests of data quality, scaling assumptions and reliability across diverse patient groups. Medical Care 32, 40-66.

[21] Mishel M (1990) Reconceptualization of the uncertainty in illness theory. Journal of Nursing Scholarship 22, 256-262.

[22] Mishel M. (1997) Uncertainty in illness scales manual Vol 1. Chapel Hill, NC university of North Carolina.

[23] NHS Strategic Health Authority (2013) Coronary artery bypass graft (CABG) surgery waiting times update. Available at: http://www.drfosterhealth.co.uk/features/cabg-waiting (accessed 6 January 2013).

[24] Rexius H, Brandrup-Wogsen G, Oden A \& Jeppsson A (2005) Waiting time and mortality after elective coronary artery bypass grafting. The annals of Thoracic Surgery 79, 538-543.

[25] Sabbah I, Drouby N, Retel-Rude N \& Mercier M (2003) Quality of life in rural and urban populations in Lebanon using SF-36 health survey. Health and Quality of Life Outcomes 1, 30-42.

[26] Sampalis J, Boukas S. Liberman M, Reid T \& Dupuis G (2001) Impact of waiting time on the quality of life of patients awaiting coronary artery bypass grafting. Canadian Medical Association Journal 165, 429-433.

[27] Screeche-Powell C \& Owen S (2003) early experiences of patients waiting to be accepted for CABG. British Journal of Nursing 12 612-619.

[28] Sobolev BG, Fradet G, Hayden R, Kuramoto L, Levy AR \& Fitzgerald MJ (2008) Delay in admission for elective coronary-artery bypass grafting is associated with increased in-hospital mortality. BMC
Health Services Research 19, 8-185. DOI: 10, 1186/1472-6963-8185. Available at: http://www.biomedcentral.com/ 147-6963/8/185.

[29] Staples P \& Jeffrey J (1997) Quality of life, hope and uncertainty of cardiac patients and their spouses before coronary artery bypass surgery. Canadian Journal of Cardiovascular Nursing 8, 7-16.

[30] Teo KK, Spoor M, Pressey T, Williamson H, Calder P, Gelfand ET $\&$ Koshal A (1998) Impact of managed waiting for coronary artery bypass graft surgery on patients' perceived quality of life. Circulation 98, 1129-1134.

[31] Williams VS, Morlock RJ \& Feltner D (2010) Psychometric evaluation of a visual analog scale for the assessment of anxiety. Health Quality of Life Outcomes 8, 8-57. DOI: 10.1186/1477-7525-8-57. 